\title{
SEARCH FOR EXTRAGALACTIC RADIO SOURCES WITH LARGE ROTATION MEASURE
}

Ko AIZU ${ }^{1}$, Makoto INOUE ${ }^{2}$, Hiroto TABARA ${ }^{3}$, and Tatsuji KATO ${ }^{3}$

${ }^{1}$ Katahira 3-24-3, Asao, Kawasaki 215, Japan

${ }^{2}$ Nobeyama Radio Observatory, Nobeyama, Minamisaku, Nagano 384-13, Japan

${ }^{3}$ Faculty of Education, Utsunomiya University, Utsunomiya 321, Japan

ABSTRACT. Intrinsic large rotation measures (RM) were searched for 128 extragalactic sources based on polarization data obtained at the NRO 45-m telescope. The number of sources with $\mathrm{RM}>500 \mathrm{rad} \mathrm{m}^{-2}$ is only seven.

Large Faraday RM observed in extragalactic radio sources is an important phenomenon for understanding an extended, hot and magnetized plasma in and around the core of QSO and active galaxies.

To find a large RM we made observations of 128 sources with a polarimeter with four channels at $10 \mathrm{GHz}$ attached to the NRO $45-\mathrm{m}$ telescope. The data obtained are combined with those by other workers and the RM is computed using the proper distribution of position angle errors, namely, Mises distribution (1918). This enables us to overcome the $n \pi$ ambiguity problem.

RMs were determined with certainty for only 40 out of 128 sources. Their distribution is shown in Fig. 1. It is clear that large RM sources are very rare. The largest four were reported by Kato et al. (1987). It is not yet clarified how this trend is related with the magnetic structure of sources.

As for the computation of $R M$ based on Mises distribution, $R M$ is determined as a local maximum of $\log ($ Likelihood):

$$
\begin{aligned}
S= & \left(\Sigma \Sigma \operatorname { c o s } \left(2 \left(\chi_{j}-\chi_{k}\right.\right.\right. \\
& \left.\left.\left.-\operatorname{RM}\left(\lambda_{j}^{2}-\lambda_{k}^{2}\right)\right)\right) \sigma_{j}^{-2} \sigma_{k}^{-2}\right)^{-1 / 2}
\end{aligned}
$$

where $\chi_{j}$ is the position angle of polarization at a wavelength $\lambda_{j}$.

\section{References}

Mises, R.v. (1918) Phys. Zeitschr. $19,490$.

Kato, T., Tabara, H., Inoue, M. and Aizu, K. (1987) Nature 329, 223.

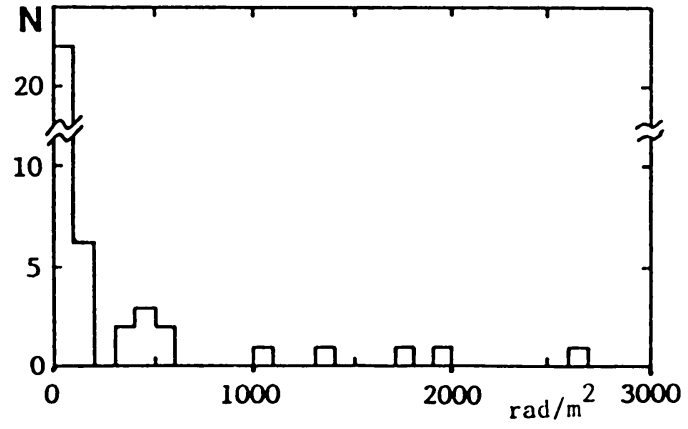

Fig. 1. Distribution of Rotation Measures of 40 sources. 472

R. Beck et al. (eds.), Galactic and Intergalactic Magnetic Fields, 472.

() 1990 IAU. Printed in the Netherlands. 